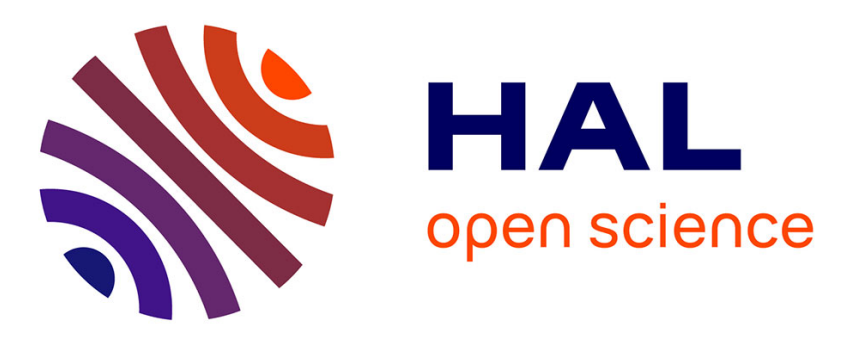

\title{
Effects of tetracycline and zinc on selection of methicillin-resistant (MRSA) Sequence Type 398 in pigs
}

Arshnee Moodley, Søren Saxmose Nielsen, Luca Guardabassi

\section{To cite this version:}

Arshnee Moodley, Søren Saxmose Nielsen, Luca Guardabassi. Effects of tetracycline and zinc on selection of methicillin-resistant (MRSA) Sequence Type 398 in pigs. Veterinary Microbiology, 2011, 10.1016/j.vetmic.2011.05.025 . hal-00724202

\section{HAL Id: hal-00724202 \\ https://hal.science/hal-00724202}

Submitted on 20 Aug 2012

HAL is a multi-disciplinary open access archive for the deposit and dissemination of scientific research documents, whether they are published or not. The documents may come from teaching and research institutions in France or abroad, or from public or private research centers.
L'archive ouverte pluridisciplinaire HAL, est destinée au dépôt et à la diffusion de documents scientifiques de niveau recherche, publiés ou non, émanant des établissements d'enseignement et de recherche français ou étrangers, des laboratoires publics ou privés. 


\section{Accepted Manuscript}

Title: Effects of tetracycline and zinc on selection of methicillin-resistant Staphylococcus aureus (MRSA) Sequence Type 398 in pigs

Authors: Arshnee Moodley, Søren Saxmose Nielsen, Luca Guardabassi

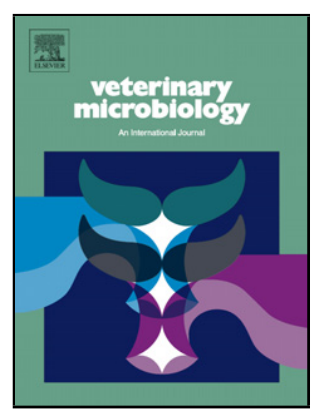

PII: S0378-1135(11)00303-8

DOI: doi:10.1016/j.vetmic.2011.05.025

Reference: VETMIC 5322

To appear in: $\quad$ VETMIC

Received date: $\quad 3-2-2011$

Revised date: $\quad 11-5-2011$

Accepted date: $\quad$ 13-5-2011

Please cite this article as: Moodley, A., Nielsen, S.S., Guardabassi, L., Effects of tetracycline and zinc on selection of methicillin-resistant Staphylococcus aureus (MRSA) Sequence Type 398 in pigs, Veterinary Microbiology (2010), doi:10.1016/j.vetmic.2011.05.025

This is a PDF file of an unedited manuscript that has been accepted for publication. As a service to our customers we are providing this early version of the manuscript. The manuscript will undergo copyediting, typesetting, and review of the resulting proof before it is published in its final form. Please note that during the production process errors may be discovered which could affect the content, and all legal disclaimers that apply to the journal pertain. 
1 Effects of tetracycline and zinc on selection of methicillin-resistant Staphylococcus 2 aureus (MRSA) Sequence Type 398 in pigs

3

4 Arshnee Moodley $^{1}$, Søren Saxmose Nielsen ${ }^{2}$ and Luca Guardabassi ${ }^{1}$

$5{ }^{1}$ Department of Veterinary Disease Biology, Faculty of Life Sciences, University of Copenhagen,

$6 \quad$ Frederiksberg C, 1870, Denmark

$7 \quad{ }^{2}$ Department of Large Animal Sciences, Faculty of Life Sciences, University of Copenhagen,

$8 \quad$ Frederiksberg C, 1870, Denmark

9

10 Running title: Selection of porcine MRSA ST398

11

12 *Corresponding author: Arshnee Moodley, Department of Veterinary Disease Biology, University of

13 Copenhagen, Stigbøjlen 4, Frederiksberg C, 1870, Denmark; Tel: +45 35332725; Fax: +45 35332757;

14 e-mail: $\underline{\text { asm@life.ku.dk }}$

15 


\section{Abstract}

17 An in vivo experiment was conducted to evaluate the effects of tetracycline and zinc on pig colonization and transmission of methicillin-resistant Staphylococcus aureus (MRSA) sequence type (ST) 398. Eight piglets naturally colonized with MRSA ST398 and 8 MRSA-negative piglets of the same age and breed were assigned to three groups treated with tetracycline and zinc (Group 1), zinc

(Group 2) or tetracycline alone (Group 3) and one non-treated group (Group 4), each containing two MRSA-positive and two MRSA-negative animals. Two additional non-treated control groups composed of only MRSA- positive (Group 5) and MRSA- negative (Group 6) animals were used to check for stability of MRSA carriage status. Nasal swabs and environmental wipes were collected on

Days $0,7,14$, and 21 , and the occurrence of MRSA in each sample was quantified by bacteriological counts on Brilliance ${ }^{\mathrm{TM}}$ MRSA agar. Significantly higher nasal MRSA counts were observed in the zinctreated $(\mathrm{p}=0.015)$ and tetracycline-treated $(\mathrm{p}=0.008)$ animals compared to the non-treated animals.

Environmental MRSA counts appeared to increase over time in Groups 1 and 2 but such an increase was not statistically significant. MRSA-negative animals housed with MRSA-positive animals became positive in all groups, whereas the carriage status of the animals in Groups 5 and 6 did not change. This study demonstrates that feed supplemented with tetracycline or zinc increases the numbers of MRSA ST398 in the nasal cavity of pigs. Transmission of MRSA from positive to negative animals housed within the same pen was not influenced by exposure to these agents.

Keywords: carriage, epidemiology, heavy metals, transmission 


\section{Introduction}

Livestock-associated MRSA belonging to sequence type (ST) 398 was first reported in the Netherlands in 2005 (Voss et al., 2005), and has spread in Europe (European Commission. 2009), Canada (Khanna et al., 2008), and in mid-western USA (Smith et al., 2009). It has been hypothesized that the emergence and spread of MRSA ST398 in pigs is driven by the use of tetracycline and zinc oxide (de Neeling et al., 2007, van Duijkeren et al., 2008, Aarestrup et al., 2010). The aim of this in vivo experiment was to assess under controlled conditions whether tetracycline and zinc, either alone or in combination, play a role in pig nasal colonization with MRSA ST398. The experiment was also used to assess the effects of the two agents on transmission from MRSA-positive to MRSA-negative animals housed within the same pen.

\section{Materials and Methods}

\section{Study design}

Eight naturally colonized MRSA-positive and 8 MRSA-negative 2-week old piglets were divided into four groups, each containing two positive and two negative animals. All treatments began after sampling on Day 0. A standard dosage of tetracycline (Terramycin ${ }^{\circledR}$ Vet, $25 \mathrm{mg} / \mathrm{kg}$ of body weight) was mixed with pig feed (DLG, Copenhagen, Denmark) and administered orally for seven days in combination with zinc (Group 1) or alone (Group 3). Zinc-oxide was mixed with the same pig feed using a standard dosage used in pig production $(2.5 \mathrm{~g} / \mathrm{kg}$ of feed) and administered orally for three weeks either alone (Group 2) or in combination with tetracycline (Group 1). Group 4 was designated as the control group and received no treatment. Two additional non-treated control groups were included in the study. Group 5 contained four 2-week old MRSA-positive piglets and was used to monitor variations in the levels of MRSA in naturally colonized animals. Group 6 contained four 2-week old 
60

61

62

63

64

65

66

67

68

69

70

71

72

73

74

75

76

77

78

79

80

81

82

MRSA-negative piglets and was used to rule out possible effects of environmental contamination on MRSA nasal carriage status. The experiment was carried out in class II isolation facilities at the Faculty of Life Sciences, University of Copenhagen, and all the procedures used were approved by the Danish National Animal Experiment Inspectorate (License no. 2006/561-1141).

\section{4}

65

\section{MRSA quantification and characterization}

Nasal swabs and environmental surfaces were collected on Days 0, 7,14 and 21. Environmental surface samples were taken from all four sides of the pen using Sodibox wipes (Sodibox, France). MRSA were semi-quantitatively assessed by bacteriological counts on Brilliance ${ }^{\mathrm{TM}}$ MRSA agar (Oxoid, United Kingdom). Nasal swabs and environmental wipes were resuspended in $1 \mathrm{~mL}$ and $100 \mathrm{~mL}$ saline, respectively, and vortexed for $15-30 \mathrm{sec}$. Thereafter, $1 \mathrm{~mL}$ of each bacterial suspension was centrifuged at $6000 \mathrm{x} g$ for $5 \mathrm{~min}$ and the pellet resuspended in $100 \mu \mathrm{L}$ of saline and spread plated on Brilliance $^{\mathrm{TM}}$ MRSA agar. Characteristic MRSA colonies (denim blue colonies with blue edges) were counted following overnight incubation at $37^{\circ} \mathrm{C}$. In parallel, the swabs were enriched in $5 \mathrm{~mL}$ Mueller Hinton broth (MHB) containing $6.5 \% \mathrm{NaCl}$ and incubated overnight at $37^{\circ} \mathrm{C}$. For samples that yielded no MRSA in the bacteriological counts, $10 \mu \mathrm{L}$ of the enrichment broth was streaked onto Brilliance ${ }^{\mathrm{TM}}$ MRSA agar and incubated overnight at $37^{\circ} \mathrm{C}$ to confirm the absence of MRSA.

One colony per Brilliance ${ }^{\mathrm{TM}}$ MRSA plate was subcultured onto 5\% blood agar and confirmed to be MRSA by mecA PCR (Zhang et al., 2004). All isolates were plated on MH agar containing $16 \mu \mathrm{g} / \mathrm{mL}$ tetracycline or 2mM zinc-chloride (Sigma-Aldrich, Denmark) to evaluate phenotypic resistance, and genes conferring tetracycline and zinc resistance was assessed by PCR (Aarestrup et al., 2000, Cavaco et al., 2010). Cfr9I-pulse-field gel electrophoresis (PFGE) (Bosch et al., 2010) was performed on all animal and environmental isolates ( $\mathrm{n}=19)$ from Group 1 to confirm transmission of MRSA between 
83 piglets and the environment within a pen. One representative of each pulsotype was further

84 characterized by spa typing (Harmsen et al., 2003).

85

86

Statistical analysis

87 The designed groups (Groups 1-4) were reorganized into treatment groups: tetracycline-treated (Groups

881 and 2) vs. non-tetracycline treated (Groups 3 and 4), and zinc-treated (Groups 1 and 3) vs. non -zinc treated (Groups 2 and 4). This was done to increase the statistical power. The counts of MRSA were compared between treatment groups using Poisson regression and taking into account the repeated structure of the data, using the Genmod procedure in SAS version 9.2 (SAS Institute, Cary, NC, USA). Nasal and environmental counts from zinc-treated, tetracycline-treated and non-treated were compared to determine if a specific treatment regimen selected for MRSA in the nasal cavity of the piglets and in the pen environment, respectively. Control groups 5 (untreated MRSA-positive piglets) and 6 (untreated MRSA-negative piglets) were not included in the statistical analysis as they were not relevant to assess the effects of treatment. This analysis was done including and excluding the counts on Day 0 to determine if the initial load of MRSA affected the MRSA counts on Days 7 to 21. Since there was no effect, and no animals received treatment prior to sampling on Day 0, counts on Day 0 were excluded from the analysis.

\section{Results}

102 Mean sample-day MRSA counts in the different treatment groups are shown in Figure 1a. Both zinctreated $(\mathrm{p}=0.015)$ and tetracycline-treated $(\mathrm{p}=0.008)$ animals had significantly higher MRSA counts in the nasal cavity of treated animals compared to non-treated animals. The average increase was in the order of 4-6 folds. Tetracycline-treated pigs had an average count of 167 colony forming units 
106 (CFU)/sampling (range: 2-1024 CFU/swab) compared to $55 \mathrm{CFU} /$ sampling (range: 0-664 CFU/swab)

107 for non-tetracycline treated pigs, and zinc-treated pigs had an average count of $221 \mathrm{CFU} / \mathrm{sampling}$

108 (range: 0-1024 CFU/swab) compared to $46 \mathrm{CFU} /$ sampling (range: 5-540 CFU/swab) in non-treated

109 pigs. No significant interaction between tetracycline and zinc was detected $(\mathrm{p}=0.62)$. The counts on

110 Days 7, 14 and 21 were not affected by the animal being positive on Day $0(\mathrm{p}=0.67)$. During the

111 experiment, MRSA counts (CFU/sampling) from environmental surfaces increased in both treatment

112 groups (Figure 1b). However, this finding was based on a limited number of observations and there was

113 no statistical association between environmental MRSA counts and treatment with zinc $(\mathrm{p}=0.09)$ or

114 tetracycline $(\mathrm{p}=0.11)$. Nasal swabs and environmental wipes from Groups 5 and 6 were MRSA-positive

115 and -negative, respectively, throughout the experiment. All samples not yielding MRSA by direct

116 selective plating on Brilliance ${ }^{\mathrm{TM}}$ MRSA agar were also negative following enrichment.

117 MRSA-negative animals housed together with MRSA-positive were positive at Day 7 and

118 remained positive for the duration of the experiment irrespective of the group to which they were

119 assigned. All isolates exhibited resistance to tetracycline and zinc as a result of the presence of two

120 tetracycline resistance genes $\operatorname{tet}(\mathrm{K})$ and $\operatorname{tet}(\mathrm{M})$, and $c z r C$, which confers resistance to zinc. The 19

121 isolates typed by PFGE had the same pulsotype and belonged to spa type t034, which is associated with

122 ST398.

123

124 Discussion

125 We demonstrated that treatment with tetracycline and zinc under controlled experimental conditions

126 results in significantly higher counts of MRSA ST398 in the nasal cavity of pigs. These results indicate

127 that either compound exerts a co-selective pressure in favour of MRSA ST398 colonization. Although

128 it may be reasonable to assume that higher levels of MRSA in the nasal cavity may facilitate animal-to- 
animal spread at the herd level, however the epidemiological significance of our results is uncertain since the efficiency of MRSA spread within farms may not be related to the numbers of nasal MRSA in individual pigs. MRSA transmission from positive to negative animals was also observed in the absence of antimicrobial selection pressure (Group 4), suggesting that short-term exposure to tetracycline and zinc is unlikely to enhance transmission. However, it cannot be excluded that prolonged use of these agents over time may result in a cumulative effect and have an impact on MRSA transmission by increasing the levels of environmental contamination.

Tetracycline is the most prescribed antibiotic for pigs in Denmark (Anonymous, 2008). Zinc is a commonly used pig feed additive in weaning piglets as it has growth promoting effects (Hill et al., 2000; Katouli et al., 1999). Heavy metals such as copper have been shown to play a role in the spread of antibiotic resistant bacteria by co-resistance i.e. presence of different resistance determinants on the same genetic element (Hasman et al., 2006). In MRSA, methicillin resistance is mediated by $m e c A$, which is carried on Staphylococcal Cassette Chromosome mec (SCCmec). Zinc oxide has the potential to co-select specifically for MRSA ST398 since $c z r C$, which encodes for cadmium and zinc resistance, is present within SCCmec elements in ST398 (Cavaco et al., 2010). Tetracycline resistance may not be a determinative factor of methicillin resistance in ST398, since tet $(\mathrm{K})$ and $\operatorname{tet}(\mathrm{M})$ are located on other mobile genetic elements (Schmitz et al., 2001, de Vries et al., 2009), and can be found in both MSSA and MRSA ST398 isolates.

A limitation of this study is the inherent difficulty in quantifying the amount of bacteria in the nasal cavity. Similar semi-quantitative approaches have been used to quantify nasal S. aureus in humans (Nouwens et al., 2004; Mermel et al., 2010). In general, high variability of the MRSA counts in individual animals was observed and such variability could be related to the effects of repeated nasal 
151 swabbing within a short period, which could interfere with the natural colonization patterns, or merely

152 reflect the natural variation in the levels of MRSA in individual animals over time.

153 In the present study we show that tetracycline and zinc, which are commonly used in pig

154 production, select for significantly higher counts of MRSA ST398 in the nasal cavity of treated pigs.

155 Although the study has some intrinsic methodological limitations concerning the semi-quantitative

156 method used for MRSA quantification and the relative short duration of the experiment, it provides

157 useful quantitative data for modelling the spread of MRSA at the herd-level, and adds novel

158 information on the relationship between antibiotic use and occurrence of resistant bacteria in animal

159 farming.

160

161 Acknowledgements

162 The study was supported by the EU FP7 PILGRIM project (Project $\mathrm{N}^{\circ}: 223050$ ). We would like to 163 acknowledge Lina M Cavaco for provision of control strains for of zinc susceptibility testing and PCR.

165 References

166 Aarestrup, F.M., Agerso, Y., Gerner-Smidt, P., Madsen, M., Jensen, L.B., 2000. Comparison of

167 antimicrobial resistance phenotypes and resistance genes in Enterococcus faecalis and Enterococcus

168 faecium from humans in the community, broilers, and pigs in Denmark. Diagn. Microbiol. Infect. Dis.

$16937,127-137$.

170 Aarestrup, F.M., Cavaco, L., Hasman, H., 2010. Decreased susceptibility to zinc chloride is

171 associated with methicillin resistant Staphylococcus aureus CC398 in Danish swine. Vet. Microbiol.

$172142,455-457$. 
Anonymous, 2008. Consumption of antimicrobial agents and occurrence of antimicrobial

174

175

176

177

178

179

180

181

182

183

184

185

186

187

188

189

190

191

192

193

194

resistance in bacteria from food animals, food and humans in Denmark. DANMAP 2008 Copenhagen,

Denmark.

Bosch, T., de Neeling, A.J., Schouls, L.M., van der Zwaluw, K.W., Kluytmans, J.A.,

Grundmann, H., Huijsdens, X.W., 2010. PFGE diversity within the methicillin-resistant

Staphylococcus aureus clonal lineage ST398. BMC Microbiol. 10, 40.

Cavaco, L.M., Hasman, H., Stegger, M., Andersen, P.S., Skov, R., Fluit, A.C., Ito, T.,

Aarestrup, F.M., 2010. Cloning and occurrence of $c z r C$, a gene conferring cadmium and zinc resistance in MRSA CC398. Antimicrob. Agents Chemother. 54, 3605-3608.

de Neeling, A.J., van den Broek, M.J., Spalburg, E.C., van Santen-Verheuvel, M.G., Dam-

Deisz, W.D., Boshuizen, H.C., van de Giessen, A.W., van Duijkeren, E., Huijsdens, X.W., 2007. High

prevalence of methicillin resistant Staphylococcus aureus in pigs.Vet. Microbiol. 122, 366-372.

de Vries, L.E., Christensen, H., Skov, R.L., Aarestrup, F.M., Agersø, Y., 2009. Diversity of the tetracycline resistance gene tet $(\mathrm{M})$ and identification of Tn916- and Tn5801-like (Tn6014) transposons in Staphylococcus aureus from humans and animals. J. Antimicrob. Chemother. 64, 490-500.

European Commission. 2009. Analysis of the baseline survey on the prevalence of methicillinresistant Staphylococcus aureus (MRSA) in holdings with breeding pigs, in the EU, 2008 [1] - Part A: MRSA prevalence estimates. Parma, Italy, 2009

(http://www.efsa.europa.eu/en/scdocs/scdoc/1376.htm) Accessed 09 July 2010

Hasman, H., Kempf, I., Chidaine, B., Cariolet, R., Ersbøll, A.K., Houe, H., Bruun Hansen, H.C., Aarestrup, F.M., 2006. Copper resistance in Enterococcus faecium, mediated by the tcrB gene, is selected by supplementation of pig feed with copper sulfate. Appl. Environ. Microbiol. 72, 5784-5789. 
Typing of methicillin-resistant Staphylococcus aureus in a university hospital setting by using novel software for spa repeat determination and database management. J. Clin. Microbiol. 41, 5442-5448.

A.J., Libal, G.W., Mahan, D.C., Shurson, G.C., Southern, L.L., Veum, T.L., 2000. Growth promotion effects and plasma changes from feeding high dietary concentrations of zinc and copper to weanling pigs (regional study). J. Anim. Sci. 78, 1010-1016.

Khanna, T., Friendship, R., Dewey, C., Weese, J.S., 2008. Methicillin resistant Staphylococcus aureus colonization in pigs and pig farmers. Vet. Microbiol. 128, 298-303.

Katouli, M., Melin, L., Jensen-Waern, M., Wallgren, P., Möllby, R., 1999. The effect of zinc oxide supplementation on the stability of the intestinal flora with special reference to composition of coliforms in weaned pigs. J. Appl. Microbiol. 87, 564-573.

Mermel, L.A., Eells, S.J., Acharya, M.K., Cartony, J.M., Dacus, D., Fadem, S., Gay, E.A., Gordon, S., Lonks, J.R., Perl, T.M., McDougal, L.K., McGowan, J.E., Maxey, G., Morse, D., Tenover, F.C., 2010. Quantitative analysis and molecular fingerprinting of methicillin-resistant Staphylococcus aureus nasal colonization in different patient populations: a prospective, multicenter study. Infect. Control Hosp. Epidemiol. 31, 592-597.

Nouwen, J.L., Ott, A., Kluytmans-Vandenbergh, M.F., Boelens, H.A.M., Hofman, A., van Belkum, A., Verbrugh, H.A. 2004Predicting the Staphylococcus aureus nasal carrier state: derivation and validation of a culture role. Clin. Infect. Dis. 39, 806-811.

Schmitz, F., Krey, A., Sadurski, R., Verhoef, J., Milatovic, D., Fluit, A.C., the European SENTRY participants., 2001. Resistance to tetracycline and distribution of tetracycline resistance genes in European Staphylococcus aureus isolates. J. Antimicrob. Chemother. 47, 239-240. 
Smith, T.C., Male, M.J., Harper, A.L., Kroeger, J.S., Tinkler, G.P., Moritz, E.D., Capuano,

A.W., Herwaldt, L.A., Diekema, D.J., 2009. Methicillin-resistant Staphylococcus aureus (MRSA)

strain ST398 is present in midwestern U.S. swine and swine workers. PLoS One. 4, e4258. van Duijkeren, E., Ikawaty, R., Broekhuizen-Stins, M.J., Jansen, M.D., Spalburg, E.C., de methicillin-resistant Staphylococcus aureus strains between different kinds of pig farms. Vet. Microbiol. 126, 383-389.

Staphylococcus aureus in pig farming. Emerg. Infect. Dis. 11, 1965-1966.

Zhang, K., Sparling, J., Chow, B.L., Elsayed, S., Hussain, Z., Church, D.L., Gregson, D.B.,

Louie, T., Conly, J.M., 2004. New quadriplex PCR assay for detection of methicillin and mupirocin resistance and simultaneous discrimination of Staphylococcus aureus from coagulase-negative staphylococci. J. Clin. Microbiol. 42, 4947-4955. 


\section{Figures legend}

233 Figure 1: a) Mean MRSA counts (CFU/sampling) from nasal swabs in the two treatment groups versus 234 the non-treated group. b) Mean MRSA counts (CFU/sampling) from environmental surface samples in 235 the two treatment groups versus the non-treated group. 

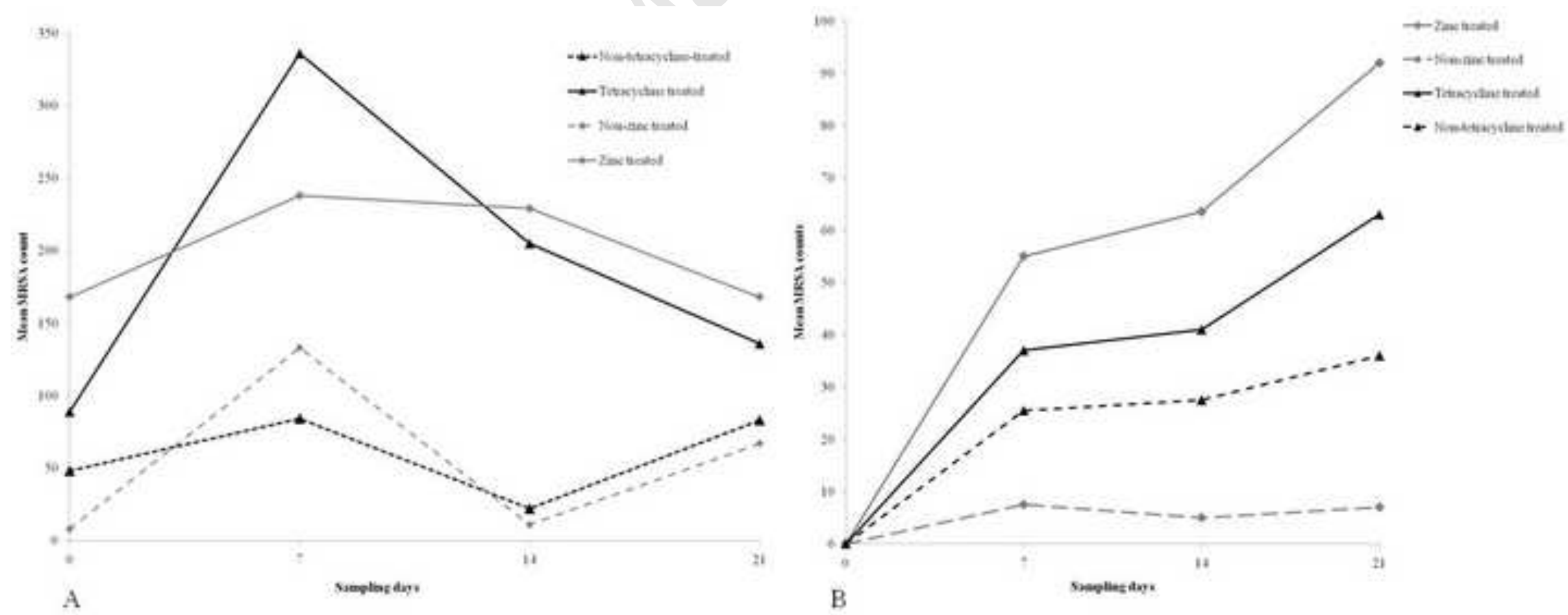